\title{
STARBURST CYCLE IN DISTANT CLUSTERS
}

\author{
A.J. BARGER, A.ARAGÓN-SALAMANCA AND R.S. ELLIS \\ Institute of Astronomy, Cambridge CB3 OHA England \\ W.J. COUCH \\ University of New South Wales, Sydney 2052 Australia
}

I. SMAIL

The Observatories of the Carnegie Institution of Washington

Pasadena, CA 91101-1292 USA

AND

R.M. SHARPLES

University of Durham, Durham DH1 $3 L E$ UK

A major puzzle in observational cosmology is the physical origin of a significant excess population of blue galaxies in the cores of distant rich galaxy clusters. This 'Butcher-Oemler' effect is now known to be a widespread starburst-related phenomenon. We test whether various spectral and photometrically-defined galaxy classes might represent different stages within a single cycle of star-formation. We compare the numbers of galaxies in various categories for three $\tilde{z}=0.31$ clusters, AC103, AC:114, and $\mathrm{AC} 118$, with evolutionary models generated according to the Bruzual \& Charlot (1993) isochrone spectral synthesis code, assuming that some fraction of the model cluster population is viewed either before or during a secondary burst of star formation. We find good agreement between the model predictions and the number density of spectroscopically-confirmed members in the $H \delta$ versus $B-R$ plane for a cluster population in which 30 per cent of the member galaxies have undergone secondary bursts of star formation within the last $\sim 2$ Gyr prior to observation. As an additional check, we analyse a larger $K_{n}$-limited sample from newly-acquired infrared images and find good agreement between the models and the data in the $U-I$ versus $I-K_{n}$ plane for the same active cluster fraction. We conclude that the unusual galaxy population in distant clusters can be explained by a single cycle in which about 30 per cent of the cluster population experienced a secondary burst of star-formation within the last $\sim 2$ Gyr. 